Article

\title{
Novel Charge Ordering in the Trimer Iridium Oxide $\mathrm{BaIrO}_{3}$
}

\author{
Ichiro Terasaki ${ }^{1}{ }^{*}$, Shun Ito ${ }^{1}$, Taichi Igarashi ${ }^{1}$, Shinichiro Asai ${ }^{1}$, Hiroki Taniguchi ${ }^{1}$, \\ Ryuji Okazaki ${ }^{2}$, Yukio Yasui ${ }^{3}$, Kensuke Kobayashi ${ }^{4}$, Reiji Kumai ${ }^{4}$, Hironori Nakao ${ }^{4}$ and \\ Youichi Murakami ${ }^{4}$
}

1 Department of Physics, Nagoya University, Nagoya 464-8602, Japan; itou.shun@d.nagoya-u.jp (S.I.); igarashi.taichi@b.mbox.nagoya-u.ac.jp (T.I.); asai.shinichiro@issp.u-tokyo.ac.jp (S.A.); hiroki_taniguchi@cc.nagoya-u.ac.jp (H.T.)

2 Department of Physics, Faculty of Science and Technology, Tokyo University of Science, Noda 278-8510, Japan; okazaki@rs.tus.ac.jp

3 Department of Physics, Meiji University, Kawasaki 214-8571, Japan; yyasui@meiji.ac.jp

4 Condensed Matter Research Center and Photon Factory, High Energy Accelerator Research Organization, Tsukuba 305-0801, Japan; kensuke.kobayashi@kek.jp (K.K.); reiji.kumai@kek.jp (R.K.); hironori.nakao@kek.jp (H.N.); youichi.murakami@kek.jp (Y.M.)

* Correspondence: terra@cc.nagoya-u.ac.jp; Tel./Fax: +81-52-789-5255

Academic Editor: Helmut Cölfen

Received: 21 December 2015; Accepted: 11 March 2016; Published: 16 March 2016

\begin{abstract}
We have prepared polycrystalline samples of the trimer $\mathrm{Ir}$ oxide $\mathrm{BaIrO}_{3}$ with face-shared $\mathrm{Ir}_{3} \mathrm{O}_{12}$ trimers, and have investigated the origin of the phase transition at $182 \mathrm{~K}$ by measuring resistivity, thermopower, magnetization and synchrotron $\mathrm{X}$-ray diffraction. We propose a possible electronic model and transition mechanism, starting from a localized electron picture on the basis of the Rietveld refinement. Within this model, $\mathrm{BaIrO}_{3}$ can be basically regarded as a Mott insulator, when the $\mathrm{Ir}_{3} \mathrm{O}_{12}$ trimer is identified to one pseudo-atom or one lattice site. The transition can be viewed as a transition from the Mott insulator phase to a kind of charge ordered insulator phase.
\end{abstract}

Keywords: face-sharing; Iridium oxide; charge order; structure-property relation

\section{Introduction}

Strongly correlated electrons are seen in a certain class of solids in which the Coulomb repulsion is too strong to hold a simple one-electron picture based on the band theory. They have occupied a central position in condensed matter sciences for many decades, and have attracted keen interests from vast numbers of researchers since the discovery of high-temperature superconducting copper oxides in 1986. They store a macroscopic number of degeneracy and entropy based on spin and charge degrees of freedom on each lattice site at high temperature [1,2], and release them through various phase transitions towards $0 \mathrm{~K}$. Various forms of phase transitions and ordered states have been discovered one after another with the progress in the studies of strongly correlated electrons. In this context, understanding of new phase transitions and ordered states is a major purpose for strong-correlation physics.

$\mathrm{BaIrO}_{3}(18 \mathrm{R}$ phase) is an interesting oxide as a playground for a new phase transition. The crystal structure is schematically shown in the inset of Figure $1 \mathrm{~b}$, in which the three $\mathrm{IrO}_{6}$ octahedra are connected with one another in a face-sharing network, and form an $\operatorname{Ir}_{3} \mathrm{O}_{12}$ trimer structure, as indicated in light brown and dark yellow. The trimers are connected with each other in a corner-sharing network and construct zig-zag chains along the $c$ axis and corrugated honeycomb lattices in the $a b$ plane. Owing to the low crystal symmetry of $C 2 / m$, two trimers are inequivalent in the unit cell, as painted in different colors in the inset of Figure $1 b$. 
The study of this oxide has a rather long history; Donohue et al. first reported the synthesis and structure of this material in the 60s, [3], and later Gai et al. [4] studied the poly-types of $5 \mathrm{H}$ and $6 \mathrm{H}$. Chamberland [5] extensively studied the relationship of the poly-types to the synthetic routes, and further found a magnetic phase transition near $200 \mathrm{~K}$. The physical and chemical properties of the poly-types have been measured rather recently because of difficulty in the sample synthesis [6,7]. Siegrist and Chamberland [8] prepared single crystals and determined the crystal symmetry of $C 2 / m$, which can be viewed as pseudo-rhombohedral $R$-centered. Later Powell and Battle [9] identified that this magnetic order is ferromagnetic, by measuring the temperature hysteresis and the magnetization-field curve. Linsay et al. [10] measured the remnant magnetization to demonstrate the ferromagnetic ground state. Cao et al. [11] measured the transport and optical properties of a single-crystal sample of $\mathrm{BaIrO}_{3}$, and found that the ferromagnetic order accompanies rapid increase in the resistivity below $T_{\mathcal{C}} \sim 180 \mathrm{~K}$. In addition, they observed a gap-like structure in the optical conductivity below around $1000 \mathrm{~cm}^{-1}$, and attributed this transition to charge-density-wave (CDW) formation with ferromagnetic order. They further found the additional two temperature anomalies near 80 and $30 \mathrm{~K}$. Although the 80-K anomaly was ill-defined, the 30-K anomaly was detected by other groups in a muon-spin-relaxation experiment [12] and in a nonlinear conduction measurement [13]. Kini et al. [14] measured the specific heat of $\mathrm{BaIrO}_{3}$ and observed a small jump of $2 \mathrm{~J} / \mathrm{mol} \mathrm{K}$ around $180 \mathrm{~K}$, not around 30 and $80 \mathrm{~K}$. They also found that the thermopower rapidly increases below $T_{c}$, and pointed out the existence of a charge gap below $T_{c}$. Maiti et al. [15] discussed the CDW state through photoemission spectroscopy.

In this paper, we try to address the nature and mechanism of the $180-\mathrm{K}$ phase transition in $\mathrm{BaIrO}_{3}$ on the basis of the electronic states constructed from a localized electron picture. A concern in the CDW scenario is that there is no evidence for the lattice distortion at $T_{c}$. In conventional CDW materials, a lattice modulation of $2 k_{F}$ emerges below $T_{c}$ through the electron-phonon interaction, where $k_{F}$ is the Fermi wavenumber [16]. To the best of our knowledge, no superlattice reflections below $T_{\mathcal{C}}$ have been reported in $\mathrm{BaIrO}_{3}$, and this implies that the charge gap opens without translational-symmetry breaking. We have investigated the structure-property relationship through synchrotron $\mathrm{X}$-ray diffraction, and propose a possible mechanism of the transition.

\section{Experimental Section}

Polycrystalline samples of $\mathrm{BaIr}_{1-x} \mathrm{Ru}_{x} \mathrm{O}_{3}(x \leq 0.1)$ were prepared using a conventional solid-state reaction. A stoichiometric mixture of powder sources of $\mathrm{BaCO}_{3}(99.9 \%), \mathrm{RuO}_{2}(99.9 \%)$ and $\mathrm{Ir}(99 \%)$ was thoroughly ground in an agate mortar. The mixture was then pre-sintered in an alumina crucible at $900{ }^{\circ} \mathrm{C}$ for $12 \mathrm{~h}$ in air, and was pressed into pellets after re-grinding. The pellets were sintered at $1000{ }^{\circ} \mathrm{C}$ for $36 \mathrm{~h}$ in air.

The synchrotron X-ray diffraction was taken at BL8A \& 8B (Photon-Factory, KEK, Tsukuba, Japan). The X-ray energy was adjusted to be $18 \mathrm{keV}$, which was carefully calibrated using a standard powder sample of $\mathrm{CeO}_{2}$. Powder samples were sealed in a silica-glass capillary of 0.1-mm diameter, and the capillary was rotated by an angle of $30^{\circ}$ from the sample-stage axis during measurement. The sample temperature was controlled using a cool helium gas or nitrogen gas. The diffraction patterns were analyzed using the Rietveld refinement with Rietan-FP code [17].

The resistivity was measured with a four-probe technique using a home-made measurement system in a liquid-helium cryostat. The thermopower was measured with a two-probe method using a home-made measurement system in a liquid-helium cryostat, and the thermopower of the measurement leads was carefully subtracted. The temperature difference was typically $0.5 \mathrm{~K}$ which was monitored with a copper-constantan differential thermocouple. The magnetization was measured with a commercial product of Quantum Design Magnetic-Property-Measurement-System. 


\section{Results}

Figure 2 shows a synchrotron $\mathrm{X}$-ray diffraction pattern of $\mathrm{BaIrO}_{3}$ at room temperature. Since the scattering intensity of oxygen is 100 times weaker than that of $\mathrm{Ba}$ and $\mathrm{Ir}$, we gave up determining the atomic position of oxygen atoms, but instead employed the values reported in the literature [8]. Then, we optimized the atomic positions of Ba and Ir atoms above $100 \mathrm{~K}$ by iterating the calculation until the $R_{\mathrm{wp}}$ and $S_{R}$ values reached less than $3 \%$ and $4 \%$, respectively. We accepted worse values of $R_{\mathrm{wp}}=3 \%-4.5 \%$ for the data below $100 \mathrm{~K}$. The experimental data are compared with the refinement at 300, 150 and $34 \mathrm{~K}$ in Figure 2b-d, respectively. As shown in Figure 2, the refinement is satisfactory; there are neither detectable impurity phases nor poly-type phases, and the deviation from the refinement seems small enough to discuss the atomic positions of the Ir atoms. We measured the diffraction patterns around $T_{\mathcal{C}}$ carefully, and found no trace of superlattice reflections. This consolidates that the phase transition does not accompany the violation of translational symmetry, also indicating that this is unlikely to be a CDW transition.

Figure 1a shows the transport properties of the polycrystalline sample of $\mathrm{BaIrO}_{3}$ obtained from the same batch as used in the X-ray measurement. The resistivity is non-metallic at all temperatures with an anomalous increase near $T_{\mathcal{C}}=182 \mathrm{~K}$. We should note that the resistivity above $T_{\mathcal{c}}$ is of activation type as indicated by the dotted line shown in the inset of Figure 1a. The slope of the dotted line gives a rough estimate of the activation gap $E_{g}^{\rho} / k_{B}$ around $240 \mathrm{~K}$, which is small in comparison with a band gap of conventional semiconductors. This is comparable to or even smaller than $k_{B} T$, and it is nontrivial whether or not we may regard this non-metallic behavior as activation-type transport. According to the band calculation of $\mathrm{BaIrO}_{3}$ [18-20], the electronic states above $T_{c}$ are metallic with no gap in the density of states.

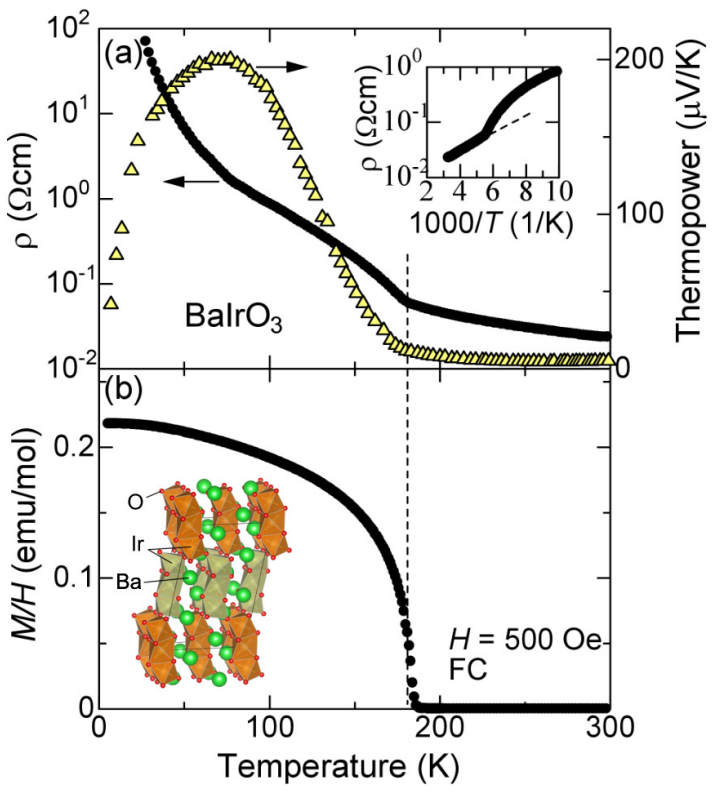

Figure 1. (a) resistivity and thermopower of a polycrystalline sample of $\mathrm{BaIrO}_{3}$. The inset shows the Arrhenius plot of the resistivity, in which the dotted line indicates the activation transport above the transition temperature $T_{c}$; (b) magnetization $M$ divided by an external field $H$ of 500 Oe of the same sample. The dotted line represents $T_{\mathcal{C}}=182 \mathrm{~K}$. The inset shows the schematic picture of the crystal structure of $\mathrm{BaIrO}_{3}$.

The thermopower also shows an anomaly near $T_{\mathcal{c}}$, below which it rapidly enhances with decreasing temperatures. As Kini et al. pointed out [14], this is a clear indication of the charge gap in the density of states [21,22], strongly indicating an insulating nature below $T_{c}$. The thermopower takes a broad maximum near $100 \mathrm{~K}$, and goes towards zero with decreasing temperature. We think 
this behavior is a crossover between the activation-type transport from 100 to $180 \mathrm{~K}$ and the hopping conduction in the impurity states below $100 \mathrm{~K}$ [23]. The thermopower above $T_{\mathcal{C}}$ is difficult to interpret; the magnitude is as small as that of metals, but the temperature dependence seems of activation type just above $T_{\mathcal{C}}$. We evaluate an activation energy $E_{g}^{S} / k_{B}$ to be $90 \mathrm{~K}$ by assuming $S=E_{g}^{S} / e T$. Again, this activation energy is comparable or smaller than $k_{B} T$. We will come back to this point later.

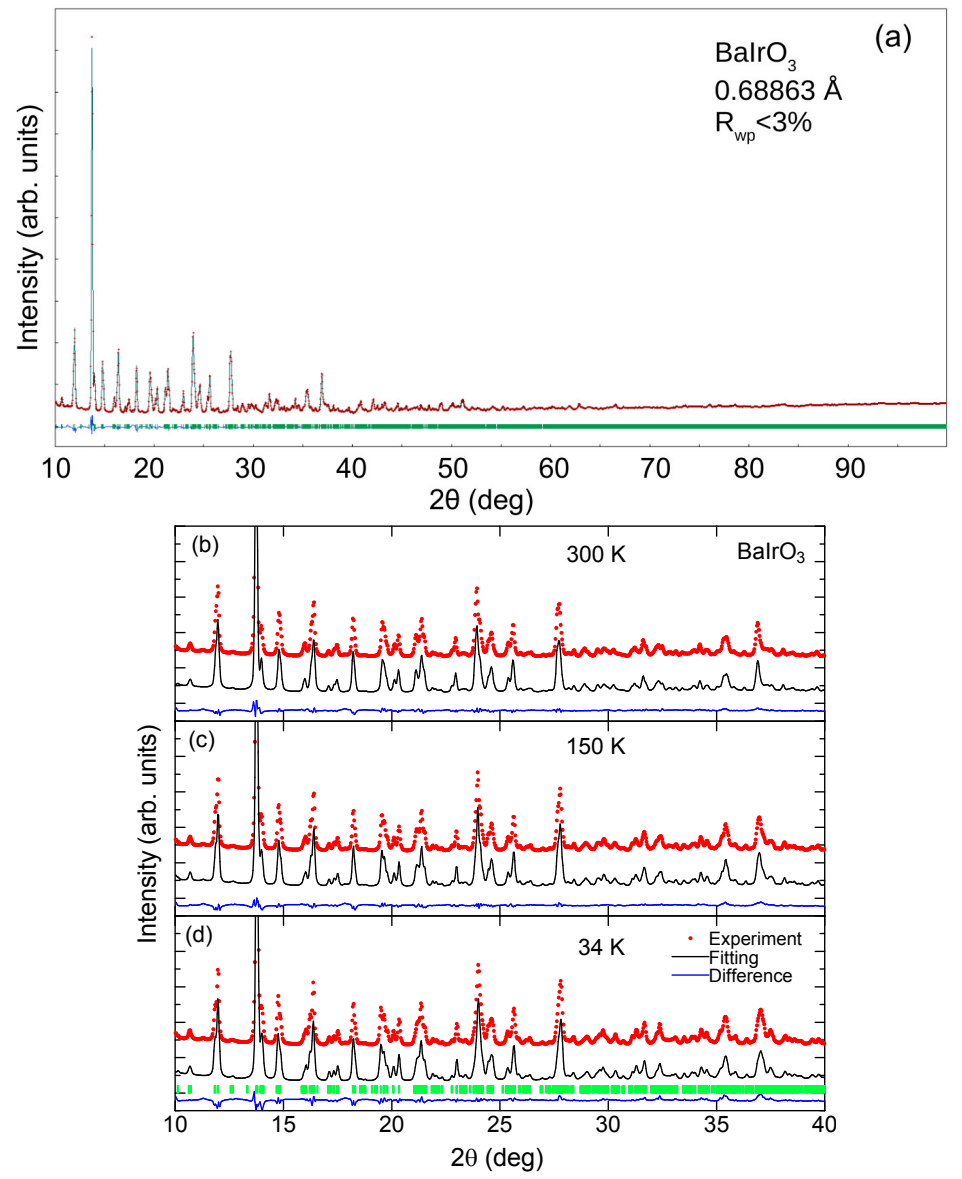

Figure 2. (a) synchrotron X-ray diffraction pattern of $\mathrm{BaIrO}_{3}$ at room temperature. For the details of the refinement, see text. Comparison between the observed data and the refinement at (b) $300 \mathrm{~K}$; (c) $150 \mathrm{~K}$; and (d) $34 \mathrm{~K}$.

Figure $1 \mathrm{~b}$ shows the magnetization divided by an external field of 500 Oe measured in the field-cooling process. Around the same temperature of $T_{C}$, the magnetization suddenly shows up with decreasing temperature. This ferromagnetic behavior is consistent with the previous reports $[10,11,24,25]$. From this figure, the magnetization at $4 \mathrm{~K}$ is evaluated to be around $100 \mathrm{emu} / \mathrm{mol}$, which corresponds to a saturation magnetization of $0.016 \mu_{B} / \mathrm{Ir}$. This unusually small moment is consistent with the previous works [10,11,24,25], and is associated with weak ferromagnetism of itinerant magnets. However, the electronic ground state of this oxide is an insulator with a finite energy gap in the density of states, and the origin of the ferromagnetism is yet to be explored.

Figure $3 a-d$ show the lattice parameters plotted as a function of temperature obtained from the Rietveld refinement of the X-ray diffraction patterns. The relative uncertainty is less than $0.01 \%$, and the size of the error bars are smaller than the size of the closed circles. All the parameters smoothly change with temperature across $T_{c}$, indicating a nature of second-order transition. By having a closer look at the data in Figure $3 \mathrm{a}$, one finds a slight cusp in the $a$-axis length near $T_{\mathcal{C}}$ as a piece of evidence for finite electron-lattice coupling. 

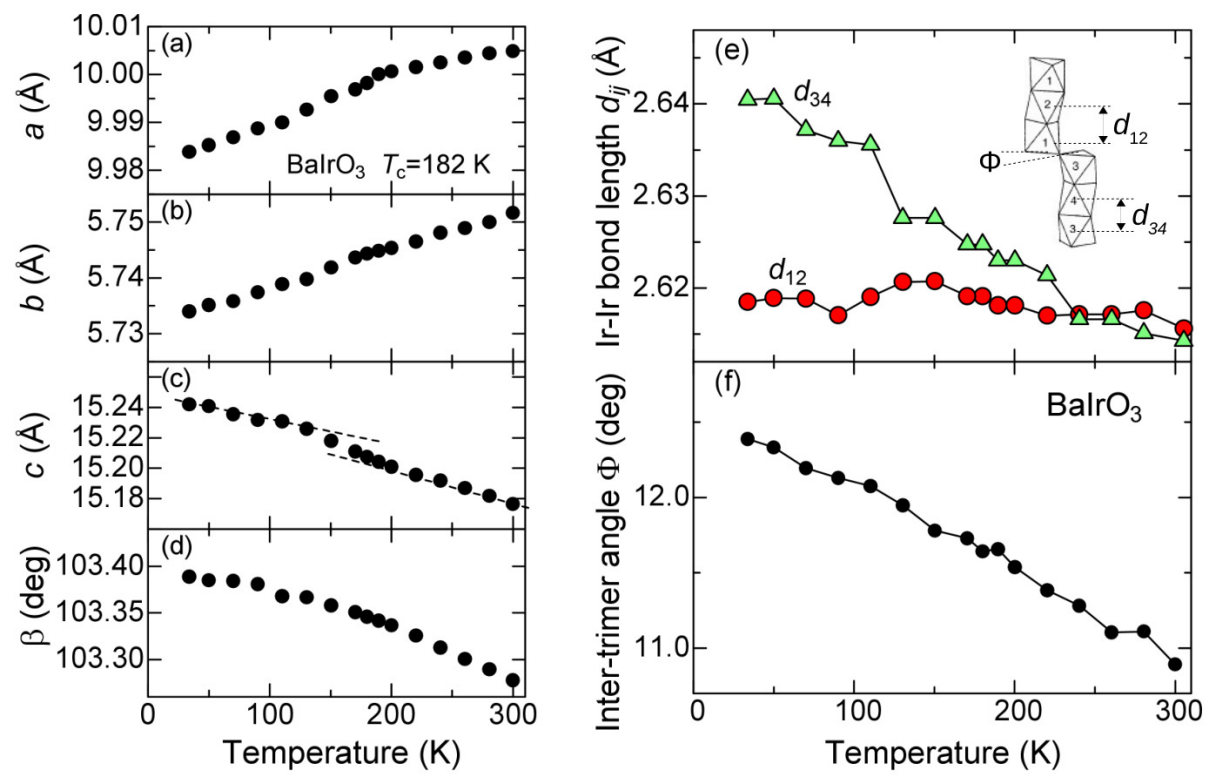

Figure 3. (a-d) lattice parameters of $\mathrm{BaIrO}_{3}$. (a) $a-$; (b) $b$-; and (c) $c$-axis length; (d) monoclinic angle $\beta$; (e) The intra-timer Ir-Ir bond length $d_{12}$ and $d_{34}$; and (f) the inter-timer angle $\Phi$. The inset schematically designates $d_{12}, d_{34}$ and $\Phi$.

The $c$-axis length parallel to the zig-zag chain of the trimers exhibits remarkable and nontrivial temperature dependence; it increases with decreasing temperature as shown in Figure 3c. Such negative temperature dependence is difficult to understand from solely lattice effects [26] except for $\mathrm{ZrW}_{2} \mathrm{O}_{8}$ [27]. The negative expansion is not observed in CDW materials [16,28], and can be another piece of evidence that this transition is not CDW-like, but, rather, the negative lattice expansion comes from various electronic origins [29]. Prime examples are Invar alloy [30], $\mathrm{BaNiO}_{3}$ [31] and $\mathrm{Mn}_{3} A \mathrm{~N}$ $(A=\mathrm{Cu}, \mathrm{Zn}, \mathrm{Ga})[32]$, in which electronic or magnetic instability tightly couples with the lattice. The slopes of the negative expansion in the $c$-axis length significantly change across $T_{c}$ as shown by the dotted lines, clearly indicating that the negative expansion characterizes the phase transition of the title compound. One may notice that the angle $\beta$ also increases with decreasing temperature. Since the increase in $\beta$ reduces the lattice volume, the $c$ axis tends to expand in spite of the volume shrinkage at low temperature.

In order to clarify the structural origin of the negative expansion in the $c$-axis length, we examined what kind of atomic displacement is responsible, and have finally arrived at the intra-trimer Ir-Ir bond length. As schematically shown in the inset of Figure 3e, there are four crystallographically inequivalent Ir sites labeled 1, 2, 3 and 4. We define the bond length between Ir1 and Ir2 as $d_{12}$ and that between Ir3 and Ir 4 as $d_{34}$. As shown in Figure $3 \mathrm{e}, d_{34}$ increases with decreasing temperature, whereas $d_{12}$ remains constant. This clearly indicates that the two timers are more inequivalent at lower temperatures. The data are somewhat scattered in comparison with the lattice parameters, possibly because the latter is determined only by the $2 \theta$ values of the diffraction peaks whereas the former needs accuracy in the relative intensity. Nevertheless, we emphasize that $d_{12}$ and $d_{34}$ were independently determined at each temperature within an uncertainty of $10^{-4}$, and that increasing inequivalence at low temperatures is inherent in this oxide. In addition, we have ascribed the structural origin for the temperature dependence of $\beta$ to the inter-timer angle $\Phi$ as indicated at the inset of Figure 3e. Figure $3 f$ shows $\Phi$ plotted as a function of temperature, where $\Phi$ increases with decreasing temperature. We can summarize the above results as follows: One trimer of Ir3-Ir4-Ir3 elongates with decreasing temperature, and concomitantly the lattice distorts to accept the elongation.

As mentioned above, it is difficult to determine the atomic position of the oxygen atoms from the synchrotron X-ray diffraction used in the present experiment, and this constraint prevents us from 
evaluating the formal valence of Ir ions using bond-valence-sum calculation. In the next section, we try to estimate the formal Ir valence by using the atomic position data for oxygen from [8]. Nevertheless, we may infer from $d_{12}$ and $d_{34}$ that the average number of $d$ electrons in the Ir3-Ir4-Ir3 timer is larger than that in the Ir1-Ir2-Ir1 trimer, for $d$ electrons feel the static Coulomb repulsion with one another to stay as far as possible. We further speculate that a finite fraction of charge rapidly transfers at $T_{c}$ between the two trimers, and also expect that this charge transfer is a driving force for the phase transition. In this respect, this charge transfer should be associated with the CDW scenario by Cao et al. [11]. One essential difference from the conventional CDW is that the two trimers are already inequivalent above $T_{\mathcal{C}}$, and this charge transfer causes no superlattice reflections with holding the same unit cell at all temperature.

\section{Discussion}

Having the above results in mind, let us propose here a possible electronic model for $\mathrm{BaIrO}_{3}$ starting from the localized picture. As schematically drawn in Figure 4a, one Ir ion has the electronic configuration of $(5 d)^{5}$ in the triply degenerate $t_{2 g}$ level, for the formal valence of Ir is $4+$. Since the oxygen octahedron is trigonally distorted, the $t_{2 g}$ level lifts the degeneracy to have the $a_{1 g}$ and doubly-degenerate $e_{g}^{\prime}$ levels. In the present case, the octahedron is compressed along the (111) direction of the local coordinate, and thus the $a_{1 g}$ level is the higher level with one electron occupied.

There is a strong hybridization between the $a_{1 g}$ levels within a trimer owing to the short intra-trimer Ir-Ir distance, and a kind of molecular orbital is expected to be formed within the trimer as shown in the right side of Figure $4 \mathrm{a}$, where we denote the bonding, non-bonding and anti-bonding orbitals made of the three $a_{1 g}$ orbitals as $\mathrm{B}, \mathrm{NB}$ and $\mathrm{AB}$, respectively. By putting the three electrons from the bottom, we find one electron in the non-bonding orbital. Thus, we may regard this state as half-filling, when we identify one trimer to one pseudo-atom. In this sense, we propose that $\mathrm{BaIrO}_{3}$ is a half-filled trimer solid.

Very recently, Ir compounds have attracted extensive interest as a strong spin-orbit interaction system, in which the total angular momentum of $j$ dominates the band structure [34]. A recent X-ray absorption study has revealed the strong spin-orbit nature in $\mathrm{BaIrO}_{3}$, and the highest occupied level should be regarded as the $e^{\prime \prime}$ level rather than the $a_{1 g}$ level from the $t_{2 g}$ levels as shown in Figure $4 \mathrm{~b}$ [33]. Even in such a case, we can make similar molecular orbitals. The highest occupied state then corresponds to the NB $e^{\prime \prime}$ instead of the NB $a_{1 g}$, and is unlikely to alter the picture of the half-filled trimer solid (compare the left and right sides of Figure 4c,d. As is widely accepted, half-filled electron systems is unstable against Mott insulator phase in the presence of electron-electron correlation. We propose the normal state of $\mathrm{BaIrO}_{3}$ is essentially in the Mott insulator phase, and name this "trimer Mott insulator".

Let us discuss here about the charge transport above $T_{c}$. According to the Landau theory of metal-insulator transition [35], there is a critical end point above which metals and insulators cannot be distinguished. In fact, the transport properties above the critical end point can be viewed as either bad metals or bad insulators, as reported in $\mathrm{V}_{2} \mathrm{O}_{3}$ [36] and $\kappa$-(BEDT-TTF) $\mathrm{Cu}\left[\mathrm{N}(\mathrm{CN})_{2}\right] \mathrm{Cl}$ [37]. We propose that the normal state of $\mathrm{BaIrO}_{3}$ should be understood basically in terms of trimer Mott insulator, with the critical end point below $T_{c}$. If so, the charge transport seems to show intermediate properties between metal and insulator, which are exemplified by the small activation energies of $E_{g}^{\rho}$ and $E_{g}^{S}$. Zhao et al. [38] have found that external pressure increases the resistivity to enhance the insulating nature of the title compound. They further found that the transition temperature decreases with pressure. Kida et al. [25] have also found the large pressure dependence of $T_{c}$ in the magnetization measurement. Their results indicate that the pressure stabilizes the insulating normal state, being consistent with our trimer-Mott-insulator picture above $T_{c}$. Cao et al. [39] have also stated that $\mathrm{BaIrO}_{3}$ is in the verge of a metallic state. 
(a)

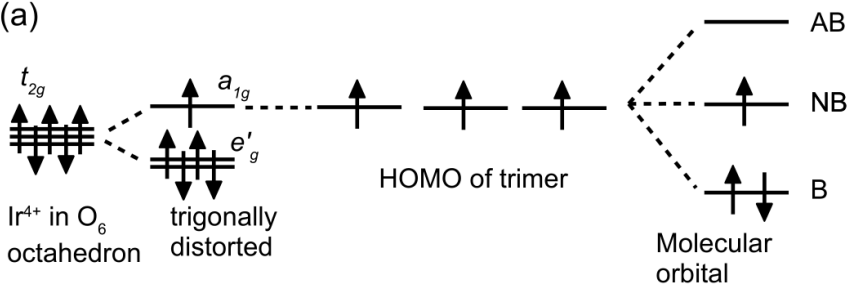

(c) $T>T_{c}$

(b)

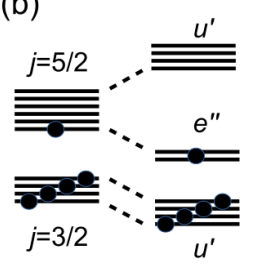

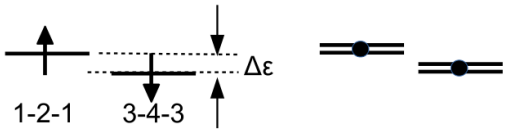

(d) $T<T_{\mathrm{c}}$

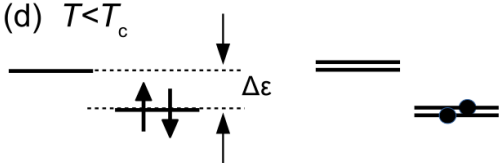

Figure 4. A model for the electronic states of $\mathrm{BaIrO}_{3}$ starting from a localized electron picture. (a) the electronic configuration of $\mathrm{Ir}^{4+}$ in a regular and trigonally distorted $\mathrm{IrO}_{6}$ (left). The highest occupied orbitals of $\operatorname{Ir}_{3} \mathrm{O}_{12}$ trimer (center) and the molecular orbital formation (right). B, NB and AB denote bonding, non-bonding and anti-bonding orbitals, respectively; (b) the electronic configuration of $\mathrm{Ir}^{4+}$ in the strong spin-orbit coupling limit (taken from [33]); (c) the electronic states of $\mathrm{BaIrO}_{3}$ above $T_{c}$. 1-2-1 and 3-4-3 correspond to the inequivalent $\operatorname{Ir}_{3} \mathrm{O}_{12}$ trimers; The case for strong spin-orbit coupling is drawn in the right side. (d) the electronic states of $\mathrm{BaIrO}_{3}$ below $T_{c}$.

Figure 4c schematically shows the trimer Mott insulator phase above $T_{c}$. The Ir1-Ir2-Ir1 trimer is in the higher level because of the shorter intra-trimer Ir-Ir bond length. All the timers have one electron in the NB $a_{1 g}$ or $e^{\prime \prime}$ level, and the energy difference $\Delta \varepsilon$ is too small to cause charge transfer. As the intra-trimer Ir-Ir bond length of the Ir3-Ir4-Ir3 trimer increases with decreasing temperature, $\Delta \varepsilon$ is expected to increase. At a certain temperature, a rapid increase of $\Delta \varepsilon$ and a charge transfer simultaneously happen, and the electronic states schematically drawn in Figure $4 \mathrm{~d}$ realize below $T_{c}$, where the NB orbital of the Ir1-Ir2-Ir1 trimer is empty and that of the Ir3-Ir4-Ir3 timer is fully occupied. From the view point of valence change, the $\mathrm{Ir}^{4+}$ state changes into a disproportionate state of $\operatorname{Ir}^{3.67+}$ and $\operatorname{Ir}^{4.33+}$ in the neighboring trimers. We regard this state as a kind of charge ordered state rather than CDW state, because this has no relation to the Fermi surface information such as nesting or $2 k_{F}$ [16]. We therefore propose that the transition at $180 \mathrm{~K}$ in $\mathrm{BaIrO}_{3}$ is a phase transition from the trimer Mott insulator phase to the trimer charge ordered phase.

We will try a rough estimate for the formal Ir valence $v$ using the bond valence sum calculation [40] given by $v=\sum_{i=1}^{6} \exp \left[\left(d_{0}-d_{i}\right) / 0.37\right]$, where $d_{i}$ is the Ir-O bond length in a $\mathrm{IrO}_{6}$ octahedron in units of angstrom, and $d_{0}=1.87 \AA$ for $\operatorname{Ir}^{4+}$ [41]. Since the positions of oxygen atoms are not well determined in the present refinement, we have instead employed the atomic position data at $300 \mathrm{~K}$ from [8] and have calculated $v$ as listed in Table 1 . The 300-K data indicate that the two trimers already have different charges; the Ir1-Ir2-Ir1 trimer has a net charge of 12.23, whereas the Ir3-Ir4-Ir3 trimer has a 0.45-shorter value of 11.78. Unlike the related ruthenate $\mathrm{Ba}_{4} \mathrm{Ru}_{3} \mathrm{O}_{10}$ [42], the charge is not disproportionated within trimer significantly. A slight difference ( 0.16 for Ir1 and Ir2; 0.07 for Ir3 and Ir4) may correspond to the bonding orbital widely spread within trimer, as is pointed out in [43]. For the calculation of the 16-K values, we used our data of $d_{12}$ and $d_{34}$ shown in Figure 3e, and assume that the trimers uniformly expand or shrink with temperature. Namely, we assume that the Ir-O bond lengths change with temperature in the same ratio as the Ir-Ir bond lengths. For the Ir3-Ir4-Ir3 trimer, $d_{34}$ increases from 2.615 to $2.640 \AA$ from 300 down to $16 \mathrm{~K}$, and thus the Ir-O bond lengths at $16 \mathrm{~K}$ equals the $300-\mathrm{K}$ value multiplied by a factor of $2.640 / 2.615=1.0096$, while they remain unchanged for the Ir1-Ir2-Ir1 
trimer. With this assumption, $v$ for Ir3 and Ir4 is further reduced from the values at $300 \mathrm{~K}$, as was discussed in the last paragraph of Section 3. At $16 \mathrm{~K}$, the Ir1-Ir2-Ir1 trimer has a net charge of 12.23, whereas the Ir3-Ir4-Ir3 trimer has a 1.05-shorter value of 11.18. This is in a reasonable agreement with the picture in Figure $4 \mathrm{~d}$ in spite of our rough and bold assumption.

Table 1. Estimation of the formal Ir valence. The atomic positions for $300 \mathrm{~K}$ are employed from [8]. For the data at $16 \mathrm{~K}$, we assume that Ir-O bond length changes with the same ratio as $d_{12}$ and $d_{34}$. Namely, we use the same Ir-O bond lengths as at $300 \mathrm{~K}$ for Ir1 and Ir2, and values $0.96 \%$-larger than at $300 \mathrm{~K}$ for Ir3 and Ir4.

\begin{tabular}{ccccc}
\hline Temperature & Ir1 & Ir2 & Ir3 & Ir4 \\
\hline $300 \mathrm{~K}$ & 4.13 & 3.97 & 3.95 & 3.88 \\
$16 \mathrm{~K}$ & 4.13 & 3.97 & 3.75 & 3.68 \\
\hline
\end{tabular}

Here, let us compare our model with band calculations reported previously [18-20]. Whangbo and Koo [18] have pointed out that the density of states of Ir2 and Ir4 (the central Ir ion in the trimer) show pseudo gap at the Fermi energy. This is explained in terms of molecular orbital. The highest occupied states belong to the non-bonding orbital of the $a_{1 g}$ or $e^{\prime \prime}$ state of the Ir ion, which has a node at the center of the trimer. Note that the non-bonding orbital is expressed by $\left(\varphi_{L}-\varphi_{R}\right) / \sqrt{2}$, where $\varphi_{L}$ and $\varphi_{R}$ are the $a_{1 g}$ or $e^{\prime \prime}$ state at the left and right edges of the trimer. The hybridization effects are also seen in other calculations $[19,20]$, and in particular, the calculation with strong spin-orbit interaction [20] gives a small Mott gap which is consistent with the observed small activation energies of $E_{g}^{\rho}$ and $E_{g}^{S}$. Since the band calculation was based on the structure at room temperature, the calculated insulating states should be those above $T_{c}$.

Our proposed model can explain various experimental results at least semi-quantitatively. It explains bad metal/bad insulator behavior above $T_{\mathcal{C}}$, a charge gap below $T_{\mathcal{C}}$ without translational-symmetry breaking, and the transition susceptible to external pressure. Since one electron in the NB $a_{1 g}$ or $e^{\prime \prime}$ state per trimer is responsible for magnetism, we can understand the small effective magnetic moment of $0.16 \mu_{B}$ per Ir above $T_{C}$ [11,24]. The transition is basically an insulator-insulator transition, which can give anomalous critical exponents [24]. This transition looks similar to the neutral-ionic transition in the charge transfer organic complexes showing non-linear conduction [44,45]. $\mathrm{BaIrO}_{3}$ also shows giant non linear conduction at low temperature [13], the origin of which may be compared with the organic complexes. Within our model, however, the weak ferromagnetism is difficult to understand, for all the trimers are nonmagnetic below $T_{c}$. The ideal charge transfer of $e$ in Figure 4c,d may not occur in real materials, but only a fraction of $e$ may move between the trimers. In such a case, all the trimers can be slightly magnetic and may show ferromagnetism with the much smaller moment of $0.016 \mu_{B}$ below $T_{c}$.

It would be fair to point out controversial points and limitations of our model. First, Figure 4 is an oversimplified picture where transfer energies except for the intra-timer case are neglected. In a real material, the complicated band structure may invalidate our model, although the band calculations reported so far do not always explain the experimental results satisfactorily. Secondly, the strong correlation, i.e., the on-site Coulomb repulsion forces the electron density to be as homogeneous as possible and disfavors the charge transfer from one trimer to another. The proposed charge order may come from a delicate balance among the on-site Coulomb repulsion, the energy difference between the two timers, and the transfer energies. A more complete theoretical modeling is needed to advance further. Third, $\mathrm{IrO}_{6}$ octahedra are distorted so that there are no pure $a_{1 g}$ and $e_{g}^{\prime}$ orbitals in a strict sense. Accordingly the molecular orbital such as NB $a_{1 g}$ may be misleading. Even in such a case, one hole on $\operatorname{Ir}^{4+}$ of $(5 d)^{5}$ occupies the non-degenerate, highly occupied orbital (say $\phi_{i}$ for Iri). Then, we can make linear combinations from $\phi_{1}$ and $\phi_{2}$ for one Ir trimer, from $\phi_{3}$ and $\phi_{4}$ for the other trimer. Again we can construct a one electron in a corresponding non-bonding-like state for one trimer, and find the essence of our model left unchanged. 
To examine the validity of our model, we have investigated the impurity effects on the $182-\mathrm{K}$ transition for a set of polycrystalline samples of $\mathrm{BaIr}_{1-x} \mathrm{Ru}_{x} \mathrm{O}_{3}$. Figure 5 shows the magnetization and the resistivity of the samples. The phase transition is systematically and drastically suppressed with the Ru concentration $x$, and essentially vanishes at only $10 \%$ substitution; For $x=0.1$, the resistivity is non-metallic without any trace of anomalies at all temperatures measured, whereas a tiny trace of the weak ferromagnetic phase of $x=0$ is visible in the magnetization measurement. The resistivity for $x=0.1$ indicates the electronic states above $T_{\mathcal{c}}$ is essentially insulating, being consistent with the picture of the trimer Mott insulator. The inset of Figure 5a shows the intra-trimer Ir-Ir bond lengths for the $x=0.1$ sample, where the two lengths of $d_{12}$ and $d_{34}$ are essentially temperature-independent, and identical. Concomitantly the negative expansion of the $c$-axis is severely suppressed (not shown). The Ru substitution simultaneously suppresses the $182 \mathrm{~K}$-transition and the electron transfer from Ir1-Ir2-Ir1 to Ir3-Ir4-Ir3, and therefore these two phenomena are two sides of the same coin. We further note that the charge order is known to be vulnerable to impurities [46], while the charge density wave is more or less robust against impurities [47].

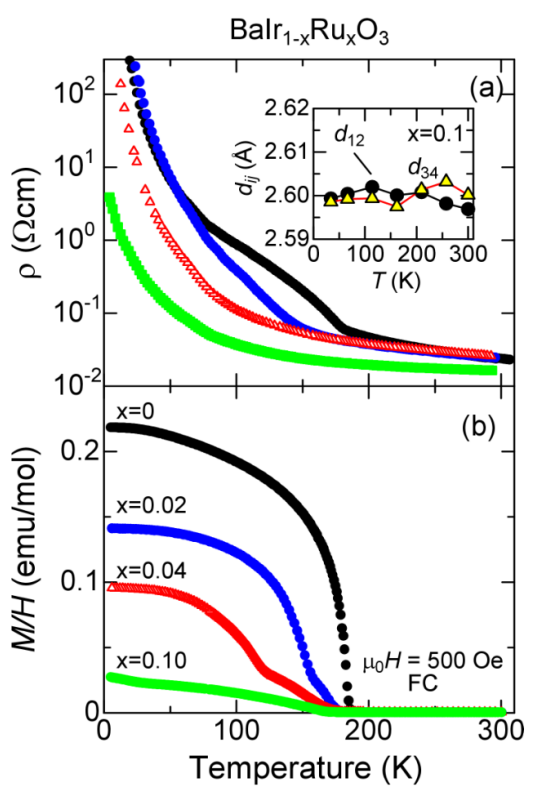

Figure 5. The impurity effect on the $182-\mathrm{K}$ transition. (a) resistivity $\rho$ of polycrystalline samples of $\mathrm{BaIr}_{1-x} \mathrm{Ru}_{x} \mathrm{O}_{3}$. The inset shows the intra-trimer Ir-Ir bond lengths for the $x=0.1$ sample. The two trimers have the identical, temperature-independent bond length; (b) magnetization $M$ divided by an external field $H$ of 500 Oe of the same samples.

A similar type of the phase transition occurs in the organic salt $\beta$-(meso-DMBEDT-TTF) ${ }_{2} \mathrm{PF}_{6}$ [48]. Many conductive organic salts with a chemical formula of $A_{2} B$ consists of a dimer molecule of $A_{2}$ as a composition unit. In the present case, two molecules of meso-DMBEDT-TTF form a dimer structure, and share one hole per dimer to behave as dimer Mott insulators [49]. Okazaki et al. [50] identified the phase transition in this compound at $70 \mathrm{~K}$ to a transition from dimer Mott insulator phase to charge ordered insulator phase. This results from a competition between the intra- and inter-dimer Coulomb interactions, which causes intrinsic inhomogeneous state detected through infrared microscope measurement. As is similar to $\mathrm{BaIrO}_{3}$, this organic salt also shows non-linear conduction below $T_{\mathcal{C}}$ [51].

Finally, we briefly comment that we have applied a similar molecular orbital concept to the related timer $\mathrm{Ru}$ oxide $\mathrm{Ba}_{4} \mathrm{Ru}_{3} \mathrm{O}_{10}$ in which the three face-shared $\mathrm{RuO}_{6}$ octahedra form a $\mathrm{Ru}_{3} \mathrm{O}_{12}$ trimer $[22,42,52]$. This related oxide shows a phase transition at $105 \mathrm{~K}$, where a metallic, paramagnetic state changes into an insulating, antiferromagnetic state at low temperature. However, the electronic 
states are different; In this related oxide, each trimer is three electrons short because $\mathrm{Ru}^{4+}$ has four $4 d$ electrons. On the basis of a similar molecular orbital approach, we have to consider partially occupied $e_{g}^{\prime}$ orbitals. In this case, the static electronic potential is deeper at the center of the trimer, and a finite fraction of charge is expected to transfer from the edge. Namely, intra-timer charge transfer drives the phase transition in $\mathrm{Ba}_{4} \mathrm{Ru}_{3} \mathrm{O}_{10}$. This makes a stark contrast to the inter-trimer charge transfer in $\mathrm{BaIrO}_{3}$. The intra-trimer charge disproportionation has been also discussed from the local-density-approximation calculations $[43,53]$.

\section{Conclusions}

In summary, we have prepared polycrystalline samples of the trimer oxide $\mathrm{BaIrO}_{3}$ with the face-shared $\mathrm{Ir}_{3} \mathrm{O}_{12}$ trimers, and have measured the resistivity, thermopower, magnetization and synchrotron X-ray diffraction from room temperature down to $4 \mathrm{~K}$. On the basis of the structure analysis, we have made a model for the electronic states of this oxide and have pointed out that this oxide can be regarded as a kind of Mott insulator, when the $\operatorname{Ir}_{3} \mathrm{O}_{12}$ trimer is identified to one lattice site. Within the same framework, the 182-K transition can be viewed as a transition from the trimer Mott insulator phase to the trimer charge-ordered phase, which is essentially different from conventional charge density wave as was used to be a candidate for the transition. We have further succeeded in explaining various properties above $T_{c}$ such as the non-metallic resistivity and thermopower, the large pressure effect, and the unusually small effective magnetic moment. We expect that the proposed concept of trimer solid will be applied to other face-shared transition metal oxides.

Acknowledgments: The authors would like to thank Mike Whangbo for showing unpublished data for the electronic states of $\mathrm{BaIrO}_{3}$. They are indebted to T. Nakano, T. Kida, M. Hagiwara, Y. Nogami, S. Mori for collaboration at an early stage of this work. They also wish to appreciate J. Akimitsu, T. Arima, S.-W. Cheong, M. Tsuchiizu for fruitful discussion and useful advice. This work was partially supported by Grant-in-Aid for Scientific Research, Japan Society for the Promotion of Science, Japan (Kakenhi No. 25610091, 26247060), and by the Program for Leading Graduate Schools “Integrative Graduate Education and Research in Green Natural Sciences", MEXT, Japan. The synchrotron X-ray diffraction was performed under the approval of the Photon Factory Program Advisory Committee (Proposal No. 2012G718 and 2012S2-005).

Author Contributions: Ichiro Terasaki conducted the whole research project and considered the electronic states of the title compound. Shun Ito made samples, and measured and analyzed all the quantities including the Rietveld refinement. Taichi Igarashi, Shinichiro Asai, Kensuke Kobayashi, Reiji Kumai, Hironori Nakao and Youichi Murakami supported the measurements of synchrotron X-ray diffraction at KEK. Hiroki Taniguchi, Ryuji Okazaki and Yukio Yasui joined the research project by mainly discussing the results and by supervising Shun Ito as a group staff member.

Conflicts of Interest: The authors declare no conflict of interest.

\section{References}

1. Furukawa, N.; Imada, M. Charge Mass Singularity in Two-Dimensional Hubbard Model. J. Phys. Soc. Jpn. 1993, 62, 2557-2560.

2. Terasaki, I.; Tsukada, I.; Iguchi, Y. Impurity-induced transition and impurity-enhanced thermopower in the thermoelectric oxide $\mathrm{NaCo}_{2-x} \mathrm{Cu}_{x} \mathrm{O}_{4}$. Phys. Rev. B 2002, 65, 195106.

3. Donohue, P.C.; Katz, L.; Ward, R. The Crystal Structure of Barium Ruthenium Oxide and Related Compounds. Inorg. Chem. 1965, 4, 306-310.

4. Gai, P.L.; Jacobson, A.J.; Rao, C.N.R. Investigation of the structure of barium iridate $\left(\mathrm{BaIrO}_{3}\right)$ by high-resolution electron microscopy. Inorg. Chem. 1976, 15, 480-483.

5. Chamberland, B. A study on the $\mathrm{BaIrO}_{3}$ system. J. Less Common Met. 1991, 171, 377-394.

6. Cheng, J.G.; Zhou, J.S.; Alonso, J.A.; Goodenough, J.B.; Sui, Y.; Matsubayashi, K.; Uwatoko, Y. Transition from a weak ferromagnetic insulator to an exchange-enhanced paramagnetic metal in the $\mathrm{BaIrO}_{3}$ polytypes. Phys. Rev. B 2009, 80, 104430.

7. Zhao, J.; Yang, L.; Yu, Y.; Li, F.; Yu, R.; Jin, C. Structural and physical properties evolution of $\mathrm{BaIr}_{1-x} \mathrm{Mn}_{x} \mathrm{O}_{3}$ solid solutions synthesized by high-pressure sintering. J. Solid State Chem. 2010, 183, 720-726.

8. Siegrist, T.; Chamberland, B. The crystal structure of $\mathrm{BaIrO}_{3}$. J. Less Common Met. 1991, 170, $93-99$. 
9. Powell, A.V.; Battle, P.D. The electronic properties of non-stoichiometric barium iridate, BaIrO $\mathrm{B}_{3-\delta}$. J. Alloys Comp. 1993, 191, 313-318.

10. Lindsay, R.; Strange, W.; Chamberland, B.; Moyer, R. Weak ferromagnetism in $\mathrm{BaIrO}_{3}$. Solid State Commun. 1993, 86, 759-763.

11. Cao, G.; Crow, J.; Guertin, R.; Henning, P.; Homes, C.; Strongin, M.; Basov, D.; Lochner, E. Charge density wave formation accompanying ferromagnetic ordering in quasi-one-dimensional $\mathrm{BaIrO}_{3}$. Solid State Commun. 2000, 113, 657-662.

12. Brooks, M.L.; Blundell, S.J.; Lancaster, T.; Hayes, W.; Pratt, F.L.; Frampton, P.P.C.; Battle, P.D. Unconventional magnetic properties of the weakly ferromagnetic metal $\mathrm{BaIrO}_{3}$. Phys. Rev. B 2005, 71, 220411.

13. Nakano, T.; Terasaki, I. Giant nonlinear conduction and thyristor-like negative differential resistance in $\mathrm{BaIrO}_{3}$ single crystals. Phys. Rev. B 2006, 73, 195106.

14. Kini, N.; Bentien, A.; Ramakrishnan, S.; Geibel, C. Specific heat and transport study of the co-existence of charge-density-wave and weak ferromagnetism in $\mathrm{BaIrO}_{3}$. Physica B 2005, 359-361, 1264-1266.

15. Maiti, K.; Singh, R.S.; Medicherla, V.R.R.; Rayaprol, S.; Sampathkumaran, E.V. Origin of Charge Density Wave Formation in Insulators from a High Resolution Photoemission Study of $\mathrm{BaIrO}_{3}$. Phys. Rev. Lett. 2005, 95, 016404.

16. Grüner, G. The dynamics of charge-density waves. Rev. Mod. Phys. 1988, 60, 1129-1181.

17. Izumi, F.; Momma, F. Three-Dimensional Visualization in Powder Diffraction. Solid State Phenom. 2007, 130, 15-20.

18. Whangbo, M.H.; Koo, H.J. Structural and electronic features of $\mathrm{BaIrO}_{3}$ causing the simultaneous occurrence of weak ferromagnetism and charge density wave formation. Solid State Commun. 2001, 118, 491-495.

19. Maiti, K. Electronic structure of $\mathrm{BaIrO}_{3}$ : A first-principles study using the local spin density approximation. Phys. Rev. B 2006, 73, 115119.

20. Ju, W.; Liu, G.Q.; Yang, Z. Exotic spin-orbital Mott insulating states in $\mathrm{BaIrO}_{3}$. Phys. Rev. B 2013, 87, 075112.

21. Sharath Chandra, L.; Lakhani, A.; Gangrade, M.; Ganesan, V. Impurity band conduction in $\mathrm{FeSi}_{1-x} \mathrm{Al}_{x}$. J. Phys. Condens. Matter 2008, 20, 325238.

22. Klein, Y.; Rousse, G.; Damay, F.; Porcher, F.; André, G.; Terasaki, I. Antiferromagnetic order and consequences on the transport properties of $\mathrm{Ba}_{4} \mathrm{Ru}_{3} \mathrm{O}_{10}$. Phys. Rev. B 2011, 84, 054439.

23. Takahashi, H.; Okazaki, R.; Yasui, Y.; Terasaki, I. Low-temperature magnetotransport of the narrow-gap semiconductor FeSb 2 . Phys. Rev. B 2011, 84, 205215.

24. Kida, T.; Senda, A.; Yoshii, S.; Hagiwara, M.; Takeuchi, T.; Nakano, T.; Terasaki, I. Unconventional critical behavior in the weak ferromagnet $\mathrm{BaIrO}_{3}$. EPL 2008, 84, 27004.

25. Kida, T.; Senda, A.; Yoshii, S.; Hagiwara, M.; Nakano, T.; Terasaki, I. Pressure effect on magnetic properties of a weak ferromagnet $\mathrm{BaIrO}_{3}$. J. Phys. Conf. Ser. 2010, 200, 012084.

26. Kittel, C. Introduction to Solid State Physics, 7th ed.; Wiley: New York, NY, USA, 1996.

27. Mary, T.A.; Evans, J.S.O.; Vogt, T.; Sleight, A.W. Negative Thermal Expansion from 0.3 to 1050 Kelvin in $\mathrm{ZrW}_{2} \mathrm{O}_{8}$. Science 1996, 272, 90-92.

28. Tian, M.; Chen, L.; Zhang, Y. Temperature dependence of the structural parameters of the host lattice in blue bronze $\mathrm{K}_{0.3} \mathrm{MoO}_{3}$. Phys. Rev. B 2000, 62, 1504-1507.

29. Takenaka, K. Negative thermal expansion materials: Technological key for control of thermal expansion. Sci. Technol. Adv. Mater. 2012, 13, 013001.

30. Colling, D.A.; Carr, W.J. Invar Anomaly. J. Appl. Phys. 1970, 41, 5125-5129.

31. Ishiwata, S.; Azuma, M.; Hanawa, M.; Moritomo, Y.; Ohishi, Y.; Kato, K.; Takata, M.; Nishibori, E.; Sakata, M.; Terasaki, I.; et al. Pressure/temperature/substitution-induced melting of $A$-site charge disproportionation in $\mathrm{Bi}_{1-x} \mathrm{La}_{x} \mathrm{NiO}_{3}(0 \leq x \leq 0.5)$. Phys. Rev. B 2005, 72, 045104 .

32. Takenaka, K.; Takagi, H. Giant negative thermal expansion in Ge-doped anti-perovskite manganese nitrides. Appl. Phys. Lett. 2005, 87, 261902.

33. Laguna-Marco, M.A.; Haskel, D.; Souza-Neto, N.; Lang, J.C.; Krishnamurthy, V.V.; Chikara, S.; Cao, G.; van Veenendaal, M. Orbital Magnetism and Spin-Orbit Effects in the Electronic Structure of $\mathrm{BaIrO}_{3}$. Phys. Rev. Lett. 2010, 105, 216407. 
34. Kim, B.J.; Jin, H.; Moon, S.J.; Kim, J.Y.; Park, B.G.; Leem, C.S.; Yu, J.; Noh, T.W.; Kim, C.; Oh, S.J.; et al. Novel $J_{\text {eff }}=1 / 2$ Mott State Induced by Relativistic Spin-Orbit Coupling in $\mathrm{Sr}_{2} \mathrm{IrO}_{4}$. Phys. Rev. Lett. 2008, 101, 076402.

35. Kotliar, G.; Lange, E.; Rozenberg, M.J. Landau Theory of the Finite Temperature Mott Transition. Phys. Rev. Lett. 2000, 84, 5180-5183.

36. Limelette, P.; Georges, A.; Jérome, D.; Wzietek, P.; Metcalf, P.; Honig, J.M. Universality and Critical Behavior at the Mott Transition. Science 2003, 302, 89-92.

37. Kagawa, F.; Itou, T.; Miyagawa, K.; Kanoda, K. Transport criticality of the first-order Mott transition in the quasi-two-dimensional organic conductor $\kappa$-BEDT-TTF ${ }_{2} \mathrm{Cu}\left[\mathrm{N}(\mathrm{CN})_{2}\right] \mathrm{Cl}$. Phys. Rev. B 2004, 69, 064511.

38. Zhao, J.; Yang, L.; Mydeen, K.; Li, F.; Yu, R.; Jin, C. Effects of pressure on electrical property of $\mathrm{BaIrO}_{3}$. Solid State Commun. 2008, 148, 361-364.

39. Cao, G.; Lin, X.N.; Chikara, S.; Durairaj, V.; Elhami, E. High-temperature weak ferromagnetism on the verge of a metallic state: Impact of dilute Sr doping on $\mathrm{BaIrO}_{3}$. Phys. Rev. B 2004, 69, 174418.

40. Brown, I.D. Recent Developments in the Methods and Applications of the Bond Valence Model. Chem. Rev. 2009, 109, 6858-6919.

41. Bond valence parameters. Available online: http://www.iucr.org/resources/data/datasets/bond-valenceparameters (accessed on 16 January 2016).

42. Igarashi, T.; Nogami, Y.; Klein, Y.; Rousse, G.; Okazaki, R.; Taniguchi, H.; Yasui, Y.; Terasaki, I. X-ray Crystal Structure Analysis and $\mathrm{Ru}$ Valence of $\mathrm{Ba}_{4} \mathrm{Ru}_{3} \mathrm{O}_{10}$ Single Crystals. J. Phys. Soc. Jpn. 2013, 82, 104603.

43. Streltsov, S.V.; Khomskii, D.I. Unconventional magnetism as a consequence of the charge disproportionation and the molecular orbital formation in $\mathrm{Ba}_{4} \mathrm{Ru}_{3} \mathrm{O}_{10}$. Phys. Rev. B 2012, 86, 064429.

44. Torrance, J.B.; Vazquez, J.E.; Mayerle, J.J.; Lee, V.Y. Discovery of a Neutral-to-Ionic Phase Transition in Organic Materials. Phys. Rev. Lett. 1981, 46, 253-257.

45. Tokura, Y.; Okamoto, H.; Koda, T.; Mitani, T.; Saito, G. Nonlinear electric transport and switching phenomenon in the mixed-stack charge-transfer crystal tetrathiafulvalene-p-chloranil. Phys. Rev. B 1988, $38,2215-2218$.

46. Tatami, N.; Ando, Y.; Niioka, S.; Kira, H.; Onodera, M.; Nakao, H.; Iwasa, K.; Murakami, Y.; Kakiuchi, T.; Wakabayashi, Y.; et al. Orbital ordering and the dilute effect in copper fluoride. J. Mag. Mag. Mater. 2007, 310, 787-789.

47. Kawabata, K. Impurity Effects on Superconductivity and Charge Density Waves in NbSe3. J. Phys. Soc. Jpn. 1985, 54, 762-770.

48. Kimura, S.; Suzuki, H.; Maejima, T.; Mori, H.; Yamaura, J.I.; Kakiuchi, T.; Sawa, H.; Moriyama, H. Checkerboard-Type Charge-Ordered State of a Pressure-Induced Superconductor, $\beta$-(meso-DMBEDT-TTF) ${ }_{2} \mathrm{PF}_{6}$. J. Am. Chem. Soc. 2006, 128, 1456-1457.

49. Kino, H.; Fukuyama, H. Electronic States of Conducting Organic $\kappa$-(BEDT-TTF) 2 X. J. Phys. Soc. Jpn. 1995, $64,2726-2729$.

50. Okazaki, R.; Ikemoto, Y.; Moriwaki, T.; Shikama, T.; Takahashi, K.; Mori, H.; Nakaya, H.; Sasaki, T.; Yasui, Y.; Terasaki, I. Optical Conductivity Measurement of a Dimer Mott-Insulator to Charge-Order Phase Transition in a Two-Dimensional Quarter-Filled Organic Salt Compound. Phys. Rev. Lett. 2013, 111, 217801.

51. Niizeki, S.; Yoshikane, F.; Kohno, K.; Takahashi, K.; Mori, H.; Bando, Y.; Kawamoto, T.; Mori, T. Dielectric Response and Electric-Field-Induced Metastable State in an Organic Conductor $\beta$-(meso-DMBEDT-TTF) ${ }_{2} \mathrm{PF}_{6}$. J. Phys. Soc. Jpn. 2008, 77, 073710.

52. Igarashi, T.; Okazaki, R.; Taniguchi, H.; Yasui, Y.; Terasaki, I. Effects of the Ir Impurity on the Thermodynamic and Transport Properties of $\mathrm{Ba}_{4} \mathrm{Ru}_{3} \mathrm{O}_{10}$. J. Phys. Soc. Jpn. 2015, 84, 094601.

53. Radtke, G.; Saúl, A.; Klein, Y.; Rousse, G. Magnetism of $\mathrm{Ba}_{4} \mathrm{Ru}_{3} \mathrm{O}_{10}$ revealed by density functional calculations: Structural trimers behaving as coupled magnetic dimers. Phys. Rev. B 2013, 87, 054436.

(C) 2016 by the authors; licensee MDPI, Basel, Switzerland. This article is an open access article distributed under the terms and conditions of the Creative Commons by Attribution (CC-BY) license (http://creativecommons.org/licenses/by/4.0/). 